\title{
Vertebroplasty versus sham procedure for painful acute osteoporotic vertebral compression fractures (VERTOS IV): randomised sham controlled clinical trial
}

\author{
Cristina E Firanescu, ${ }^{1}$ Jolanda de Vries, ${ }^{1,2}$ Paul Lodder, ${ }^{2}$ Alexander Venmans, ${ }^{1}$ \\ Marinus C Schoemaker, ${ }^{1}$ Albert J Smeet, ${ }^{1}$ Esther Donga, ${ }^{1}$ Job R Juttmann, ${ }^{1}$ Caroline A H Klazen ${ }^{3}$ \\ Otto E H Elgersma, ${ }^{4}$ Frits H Jansen, ${ }^{5}$ Alexander V Tielbeek, ${ }^{5}$ Issam Boukrab, ${ }^{1}$ \\ Karen Schonenberg, ${ }^{1}$ Willem Jan J van Rooij, ${ }^{1}$ Joshua A Hirsch, ${ }^{6}$ Paul N M Lohle ${ }^{1}$
}

${ }^{1}$ Departments of Radiology and Internal Medicine, Elisabeth TweeSteden Hospital, Hilvarenbeekseweg 60, 5022 GC, Tilburg, Netherlands

${ }^{2}$ Department of Medical and Clinical Psychology, Tilburg University, Tilburg, Netherlands ${ }^{3}$ Department of Radiology, Medisch Spectrum Twente, Enschede, Netherlands

${ }^{4}$ Department of Radiology, Albert Schweitzer Hospital, Dordrecht, Netherlands

${ }^{5}$ Department of Radiology, Catharina Hospital, Eindhoven, Netherlands

${ }^{6}$ Department of Radiology, Massachusetts General Hospital, MA, Boston, USA Correspondence to:

CE Firanescu

crisfiranescu@hotmail.com

Additional material is published online only. To view please visit the journal online.

Cite this as: $B M J$ 2018;360:k1551 http://dx.doi.org/10.1136/bmj.k1551

Accepted: 19 March 2018

\section{ABSTRACT}

OBJECTIVE

To assess whether percutaneous vertebroplasty results in more pain relief than a sham procedure in patients with acute osteoporotic compression fractures of the vertebral body.

\section{DESIGN}

Randomised, double blind, sham controlled clinical trial.

\section{SETTING}

Four community hospitals in the Netherlands, 2011-15. PARTICIPANTS

180 participants requiring treatment for acute osteoporotic vertebral compression fractures were randomised to either vertebroplasty $(n=91)$ or a sham procedure $(n=89)$.

\section{INTERVENTIONS}

Participants received local subcutaneous lidocaine (lignocaine) and bupivacaine at each pedicle. The vertebroplasty group also received cementation, which was simulated in the sham procedure group.

\section{MAIN OUTCOME MEASURES}

Main outcome measure was mean reduction in visual analogue scale (VAS) scores at one day, one week, and one, three, six, and 12 months. Clinically significant pain relief was defined as a decrease of 1.5 points in VAS scores from baseline. Secondary outcome measures were the differences between groups for changes in the quality of life for osteoporosis and Roland-Morris disability questionnaire scores during 12 months' follow-up. RESULTS

The mean reduction in VAS score was statistically significant in the vertebroplasty and sham procedure groups at all follow-up points after the procedure

\section{WHAT IS ALREADY KNOWN ON THIS TOPIC}

Owing to conflicting trial results the role of vertebroplasty in treating acute osteoporotic compression fractures of the vertebral body is the subject of debate

\section{WHAT THIS STUDY ADDS}

Percutaneous vertebroplasty to treat patients with acute osteoporotic vertebral compression fractures did not result in statistically significant more pain relief than a sham procedure during 12 months' follow-up

The results of this trial do not support percutaneous vertebroplasty as standard pain treatment in patients with acute osteoporotic vertebral compression fractures compared with baseline. The mean difference in VAS scores between groups was $0.20(95 \%$ confidence interval -0.53 to 0.94$)$ at baseline, $-0.43(-1.17$ to $0.31)$ at one day, $-0.11(-0.85$ to 0.63$)$ at one week, $0.41(-0.33$ to 1.15$)$ at one month, $0.21(-0.54$ to $0.96)$ at three months, 0.39 ( -0.37 to 1.15$)$ at six months, and 0.45 ( -0.37 to 1.24 ) at 12 months. These changes in VAS scores did not, however, differ statistically significantly between the groups during 12 months' follow-up. The results for secondary outcomes were not statistically significant. Use of analgesics (non-opioids, weak opioids, strong opioids) decreased statistically significantly in both groups at all time points, with no statistically significant differences between groups. Two adverse events occurred in the vertebroplasty group: one respiratory insufficiency and one vasovagal reaction.

\section{CONCLUSIONS}

Percutaneous vertebroplasty did not result in statistically significantly greater pain relief than a sham procedure during 12 months' follow-up among patients with acute osteoporotic vertebral compression fractures.

TRIAL REGISTRATION

ClinicalTrials.gov NCT01200277.

\section{Introduction}

Osteoporotic compression fractures of the vertebral body can result in pain and long term morbidity, including spinal deformity. Conservative management includes opioids and other analgesics, bed rest, and a back brace. In percutaneous vertebroplasty, polymethylmethacrylate is injected into the fractured vertebral body; a technique that became widespread on the basis of preliminary observational and trial results $^{1-3}$ and was endorsed by professional societies. ${ }^{4}$ In 2009 two blinded randomised studies found no short term benefit of vertebroplasty compared with sham controls. ${ }^{56}$ In 2010, an open label randomised controlled trial, VERTOS II (vertebroplasty versus conservative treatments in acute osteoporotic vertebral compression fractures II), showed that vertebroplasty resulted in pain reduction, more rapid pain relief, more pain-free days, better functional outcome, and improved quality of life. ${ }^{7}$ In 2016 the VAPOUR (vertebroplasty for acute painful osteoporotic fractures) trial showed that better pain relief was achieved after vertebroplasty compared with sham treatment, as measured by a score of less than 4 on a visual analogue 
scale (VAS) at 14 days, improved quality of life, and $30 \%$ greater preservation of vertebral body height. ${ }^{8}$

We clarified the role of percutaneous vertebroplasty in the treatment of acute osteoporotic vertebral compression fractures using a double blind randomised controlled trial with a sham control. With this trial design we intended to improve on limitations of the 2009 trials ${ }^{5}{ }^{6}$ by using stricter inclusion and exclusion criteria, larger patient cohorts, longer clinical and imaging follow-up, and no crossovers.

\section{Methods}

Study design

This trial was a sham controlled, double blind multicentre randomised trial performed at four community hospitals in the Netherlands. All participants gave written informed consent to take part in the trial.

The primary objective was to determine the difference between groups in mean reduction of scores on a VAS during 12 months' follow-up at one day, one week, and one, three, six, and 12 months after the procedure. The secondary objective was to determine the differences between groups in mean reduction of quality of life and physical functioning at one week and one, three, six, and 12 months. This study was limited specifically to acute osteoporotic vertebral compression fractures, along with bone oedema on magnetic resonance imaging. ${ }^{7} 8$ We did not allow crossovers during follow-up. Participants were informed about the importance of remaining in the same treatment arm for 12 months and verbally agreed to this request. Participants in the percutaneous vertebroplasty group underwent computed tomography immediately after the procedure to check for cement leakage; this was simulated in the sham procedure group to protect the participants from unnecessary exposure to ionising radiation and to blind them to their treatment allocation. Spine radiographs were obtained at three, six, and 12 months.

\section{Participants}

Inclusion criteria were age 50 years or more, 1-3 vertebral compression fractures, T5-L5 focal back pain at the level of fracture for up to six weeks, score of 5 or higher on a VAS, diminished bone density ( $\mathrm{T}$ score -1 or less) on a dual energy $\mathrm{x}$ ray absorptiometry (DEXA) scan, 15\% or more loss of vertebral height, and bone oedema on magnetic resonance imaging. Owing to difficulties with recruitment, six months after initiating recruitment we also included patients with pain up to nine weeks. An internist performing physical examinations provided independent confirmation of focal pain with a score of 5 or higher on a VAS at the fracture site before randomisation. Exclusion criteria were severe cardiopulmonary morbidity, untreatable coagulopathy, systemic or local spine infection, suspected malignancy, neurological symptoms, or inability to undergo magnetic resonance imaging. A detailed review of the protocol has been published previously and can be accessed online. ${ }^{9}$

\section{Randomisation and blinding}

Participants were randomised by computer in a block size of six, randomisation ratio $1: 1$, and a maximum sample size of 84 for each participating centre. Participants, internists, and outcome assessors were blinded and remained so during the 12 months' followup. It was not possible to mask the interventional and diagnostic radiologists.

\section{Procedures}

All participants received local infiltration with $1 \%$ lidocaine (lignocaine) into each pedicle followed by $0.25 \%$ bupivacaine. Each participant received two stab incisions at the level of the vertebral body, after which the sealed randomisation envelope was opened. Bone biopsy needles were positioned bilaterally, using standard transpedicular placements for vertebroplasty or periosteal placements for the sham procedure. Participants in the vertebroplasty group were exclusively treated with local anaesthetic unless they required conscious sedation (50 $\mu \mathrm{g}$ fentanyl in $22 \%(n=20)$ of patients). In both groups the cement (polymethylmethacrylate) was prepared in close proximity to the participants. As a result, the mixing sound could be heard and the polymethylmethacrylate smelt by everyone in the room. In the vertebroplasty group, a cement injector was attached to the previously placed needles. In the sham procedure group we simulated the injection phase using verbal and physical cues. In the vertebroplasty group, polymethylmethacrylate injection was stopped when cement leakage was noticed. In participants with multiple fractures, all were treated according to the assigned group. Additional analgesics were allowed for participant comfort during the procedure. After the procedure, participants in the vertebroplasty group underwent computed tomography, whereas participants in the sham procedure group were subjected to preliminary scout view scanning only. We defined the threshold for leakage as anything perceptible on computed tomography.

All participants were outpatients. Because participants and nurses were blinded to the intervention, both study groups received the same patient care during hospital stay and at discharge. Participants were advised to restart daily activities after discharge. Drugs for osteoporosis were prescribed according to the standard protocol of the participating institutions for patients with osteoporotic vertebral fractures.

\section{Outcomes}

Participants were assessed at baseline (day of the procedure), one day, one week, and one, three, six, and 12 months after the procedure. Analgesic treatment was modified when appropriate at intake or follow-up. We categorised the analgesics according to the World Health Organization classification as no drugs, non-opioids, weak opioids, and strong opioids. ${ }^{10}$ The interventional radiologist recorded complications and adverse events during the 
procedure and until discharge. Adverse events were recorded as written reports. Spine radiographs were obtained at baseline and at three, six, and 12 months. Two independent diagnostic radiologists obtained visual semiquantitative scoring and morphometric measurements of every vertebral compression fracture. Assessment of vertebral fractures was based on the Genant classification. ${ }^{11}$ To avoid measurement errors, no magnifications were used. We defined bone oedema in the vertebral compression fracture as increased signal intensity on short tau inversion recover (STIR) magnetic resonance images and decreased signal intensity on the T1 weighted images. Vertebral osteonecrosis was defined as a well circumscribed area of low signal intensity on T1 weighted magnetic resonance images, with high signal intensity on T2 weighted images.

The primary prespecified objective was to determine the difference between groups in mean reduction of VAS score during 12 months' follow-up, at one day, one week, and one, three, six, and 12 months after the procedure. The VAS score ranged from 0 (no pain) to 10 (severe pain). ${ }^{12}$ We defined clinically significant pain relief as a decrease of 1.5 points on the VAS from baseline in each group. ${ }^{7}$ The secondary prespecified objective was to determine differences between groups in changes of quality of life and physical functioning during 12 months' follow-up, at one week and one, three, six, and 12 months after the procedure. We measured quality of life with the quality of life questionnaire of the European Foundation for Osteoporosis (QUALEFFO) ${ }^{13}$ and physical functioning with the modified Roland-Morris disability (RMDQ) questionnaire. ${ }^{14}$ The QUALEFFO score is based on 41 questions and is calculated as a sum of all answers to items, which are then linearly transformed on the scale $0-100$. The worse the health related quality of life condition, the higher the score. The anchors vary between scales as well as between questions within a scale. An example of an anchor for the scale pain is "no back pain" to "unbearable" (5 point response scale). Another example of the scale activities of daily living is "no difficulty" to "impossible without help" (5 point response scale). The RMDQ has 24 items that assess functional status over the past 24 hours in patients with back pain. This questionnaire does not have response scales but only the instruction: When you read a sentence that describes you today, mark the box next to it. The RMDQ has a range of 0 (no disability due to back pain) to 24 (maximum disability due to back pain), with higher scores indicating higher disability from back pain.

Post hoc analysis included the proportion of patients in both groups at 12 months' follow-up with a VAS score of 5 or higher, progressive loss of vertebral body height of treated fractures (defined as $\geq 4 \mathrm{~mm}$ ), and a new fracture. ${ }^{15}$ Treatment of new fractures was according to the initial assignment and the same inclusion criteria. We did not allow crossovers. One day after the intervention we asked participants to guess which procedure they had undergone.

\section{Statistical analysis}

Analyses were performed using IBM SPSS statistics 23 and the R programming software (R development Core Team, 2008). ${ }^{16}$ We considered $\mathrm{P}$ values $<0.05$ to indicate statistical significance. G-Power was used to calculate the sample size. Assuming a 1.5 point difference on the VAS between the groups, a pooled standard deviation of 4.23, an effect size of 0.18 , and $20 \%$ withdrawal $(\alpha=0.05$ and $\beta=0.20$ at seven time points), we determined that we needed 90 patients in each group..$^{5-7} 1718$ Analysis was by intention to treat-that is, we analysed patients on the basis of their initial treatment assignment. We used $\chi^{2}$ tests to compare the proportions of adverse events, drugs, and baseline fractures. For continuous and normally distributed variables, we computed means and standard deviations, whereas for nonnormally distributed variables we report the medians and interquartile ranges. We used a mixed modelling approach to analyse our primary endpoint (difference between groups in mean reduction of VAS score during 12 months' follow-up) and secondary endpoints (difference between groups in mean reduction of QUALEFFO and RMDQ during 12 months' follow-up). The statistical interaction between treatment and time was used to evaluate the primary and secondary endpoints. By making use of the custom hypothesis test command in the SPSS syntax we derived tests directly from the mixed model for differences between the groups at specific time points. A mixed model analysis makes use of all available data for each participant, thereby accounting for data missing at random. Model estimates were adjusted based on the covariates age, sex, vertebral level, pain treatment, osteonecrosis, new fractures after baseline, and progressive loss of vertebral height. We used a first order autoregressive covariance structure to model the repeated measurements. Cox regression analysis was used to determine the extent to which patients reached statistically significant pain relief over time. We also carried out exploratory post hoc analyses to determine whether progressive loss of vertebral height interacted with treatment in predicting the pain intensity during 12 months' follow-up. We used $\chi^{2}$ tests to assess the difference between groups in the occurrence of new fractures and to examine the difference in proportion of patients in each group with a VAS score of 5 or higher at 12 months' follow-up. To investigate differences between groups in the use of analgesics during 12 months' follow-up we used generalised mixed modelling.

\section{Patient involvement}

No patients were involved in setting the research question or the outcome measures, nor were they involved in developing plans for recruitment, design, or implementation of the study. No patients were asked to advise on interpretation or writing up of results. There are no plans to disseminate the results of the research to study participants or the relevant patient community. 


\section{Results}

\section{Patient characteristics}

Of 1280 patients with focal back pain and one to three thoracolumbar fractures screened for eligibility, 180 were randomly allocated: 91 to the vertebroplasty group and 89 to the shame procedure. Overall, 156 eligible patients declined to participate for several reasons, some of which included treatment with open label vertebroplasty. After randomisation, four patients were excluded for reasons ranging from unexpected malignancy to missing intake form. These patients were not included in the intention to treat analysis, resulting in 90 participants in the vertebroplasty group and 86 in the sham procedure group. Over 12 months' follow-up, 13 patients (eight vertebroplasty, five sham procedure) died of unrelated causes (fig 1). Twenty two of the 90 participants (24\%) in the vertebroplasty group and 12 of the 86 participants (14\%) in the sham procedure group had pain for more than six weeks and less than nine weeks before randomisation.

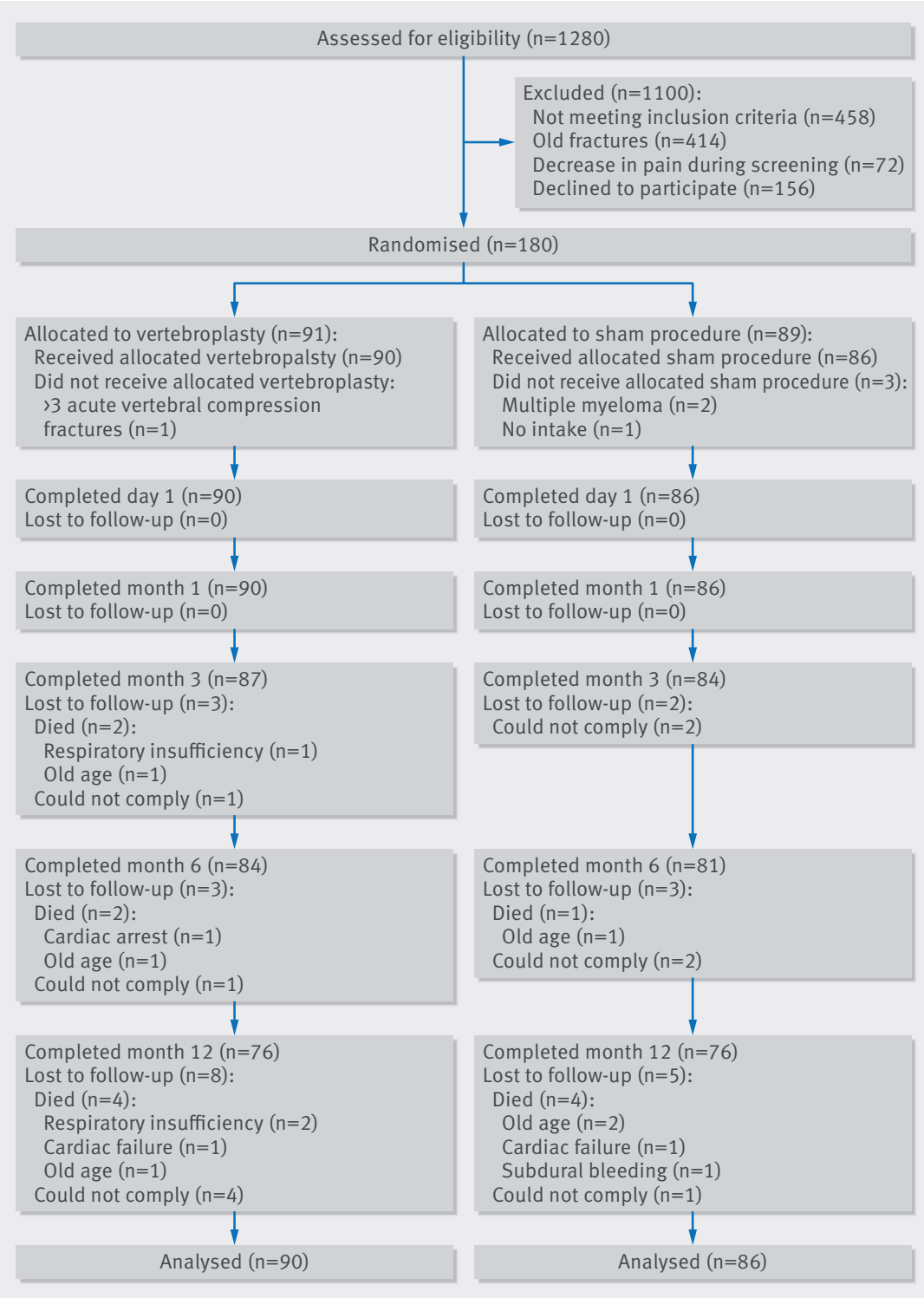

Fig 1 | Flow of participants through study
Recruitment was slow, mostly because patients did not meet the inclusion criteria or had older fractures.

Ninety patients with 115 fractures underwent vertebroplasty and 86 patients with 108 fractures underwent the sham procedure. Three or fewer fractures were treated in a single session in each cohort. In the vertebroplasty group only one vertebral compression fracture was present in 72 participants, two in 15 participants, and three in two participants. In the sham procedure group only one vertebral compression fracture was present in 64 participants, two in 15 participants, and three in seven participants.

The day after treatment, $82 \%(n=74)$ of participants in the vertebroplasty group and $81 \%(n=70)$ in the sham procedure group believed they had undergone cementation. The remainder were either unsure or thought they had undergone the sham procedure. The median duration between the onset of focal back pain and initial radiography was 30 days (interquartile range 15-42 days) in the vertebroplasty group and 23 (15-36) days in the sham procedure group. After initial radiography, vertebroplasty occurred at a median of 13 days (interquartile range 7-18 days) and the sham procedure at a median of 11 (7-17) days. The median time from onset of symptoms to treatment was 43 days (interquartile range 29-52 days) in the vertebroplasty group and 36 (24-51) days in the sham procedure group. Both groups had similar baseline characteristics (table 1).

\section{Primary outcome}

Compared with baseline, the reduction in VAS score during 12 months in both groups was clinically and statistically significant at all measurement points (one day, one week, and one, three, six, and 12 months). This significant reduction was measurable starting at day 1 after the procedure and remained significant throughout follow-up. The mean VAS score declined at the last measurement point (12 months), by 5.00 in the vertebroplasty group (95\% confidence interval 4.31 to 5.70) and by 4.75 in the sham procedure group (3.93 to 5.57). However, a non-significant interaction between treatment and time showed that these reductions in VAS scores did not differ between the groups $(F(6$, 838.66)=0.92, $\mathrm{P}=0.48)$. The difference between groups for change in VAS score between baseline and 12 months was 0.13 (95\% confidence interval -0.41 to 0.66$)$. The difference between the groups was not statistically significant at one day, one week, and one, three, six, and 12 months after the procedure (fig 2 and table 2).

\section{Secondary outcomes}

A similar pattern of improvement was observed for the QUALEFFO and RMDQ outcomes. In both groups the reduction in QUALEFFO and RMDQ score achieved at one week and one, three, six, and 12 months was statistically significant compared with baseline. However, on both outcomes the groups did not differ in change over time (QUALEFFO: $F(5,786.56)=1.37$, $\mathrm{P}=0.24$; RMDQ: $F(6,748.32)=1.55, \mathrm{P}=0.17)$. The mean reduction in QUALEFFO score at the last measurement 


\begin{tabular}{|c|c|c|}
\hline Characteristics & Vertebroplasty $(n=90)$ & Sham procedure $(n=86)$ \\
\hline Mean (SD) age (years) & $74.7(10.7)$ & $76.9(8.1)$ \\
\hline Median (interquartile range) No of days with back pain before procedure & $43(29-52)$ & $36(24-51)$ \\
\hline Median (interquartile range) No of days from radiographic diagnosis to procedure & $13(7-18)$ & $11(7-17)$ \\
\hline No of vertebral compression fractures at baseline & 115 & 108 \\
\hline Mild (20-25\%) & $37(32)$ & $30(28)$ \\
\hline Moderate (25-40\%) & $51(44)$ & $49(45)$ \\
\hline Severe $(>40 \%)$ & $27(23)$ & $30(28)$ \\
\hline Wedge & $56(49)$ & $65(60)$ \\
\hline Biconcave & $59(51)$ & $44(40)$ \\
\hline Strong opioid derivatives & $42(47)$ & $25(29)$ \\
\hline \multicolumn{3}{|l|}{ Vertebral level with bone oedema: } \\
\hline $\mathrm{T} 5-\mathrm{T} 10$ & $36(31)$ & $24(22)$ \\
\hline $\mathrm{T} 11-\mathrm{L} 2$ & $59(51)$ & $69(64)$ \\
\hline L3-L5 & $20(17)$ & $15(14)$ \\
\hline \multicolumn{3}{|l|}{ No of spinal levels treatedt: } \\
\hline 1 & $70(78)$ & $67(78)$ \\
\hline 2 & $15(17)$ & $15(17)$ \\
\hline 3 & $5(6)$ & $4(5)$ \\
\hline Drugs for osteoporosis & $42(47)$ & $49(57)$ \\
\hline
\end{tabular}

point (12 months) was 18.32 in the vertebroplasty group (95\% confidence interval 18.32 to 23.61 ) and 18.61 in the sham procedure group (13.02 to 24.2), with a between group difference of -0.14 (95\% confidence interval -3.04 to 2.76 ). The mean reduction in RMDQ score at the last measurement point (12 months) was 7.71 in the vertebroplasty group (5.87 to 9.55 ) and 7.47 in the sham procedure group (5.56
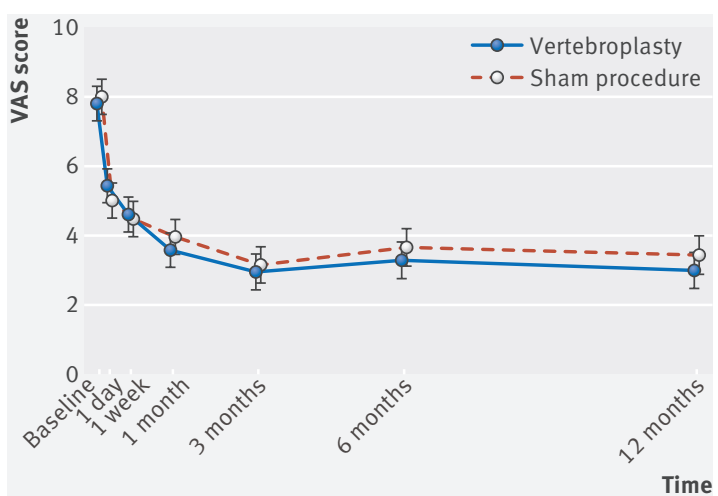

Fig 2 | Mean visual analogue scale (VAS) score for participants in vertebroplasty and sham procedure groups during 12 months' follow-up. Whiskers represent $95 \%$ confidence intervals to 9.38), with a between group difference of 0.12 (-1.11 to 1.35). Appendix tables A1 and A2 show no statistically significant differences between groups at all follow-up points after the procedure.

\section{Ancillary analyses}

A post hoc analysis showed that at 12 months' follow-up a significantly higher percentage of participants in the sham procedure group $(41 \% ; n=30)$ had VAS scores of 5 or higher compared with participants in the vertebroplasty group $(20 \% ; \mathrm{n}=16):\left(\chi^{2}(1)=8.08, \mathrm{P}=0.005\right.$, odds ratio $0.36,95 \%$ confidence interval 0.17 to 0.74 ).

Figure 3 depicts the proportion of participants who reached the patient satisfaction level of clinically significant pain relief during follow-up. A Cox regression analysis showed no statistically significant differences in pain relief between the two groups at the 12 month follow-up after adjusting for the clinically important covariate strong opiate intake (hazard ratio 1.02 , 95\% confidence interval 0.86 to 1.21 , $\operatorname{Wald}(1)=0.06, \mathrm{P}=0.80)$.

Another non-prespecified analysis showed that during follow-up significantly more participants in the sham procedure group $(45 \%, n=39)$ had progressive loss of height in treated vertebrae compared with participants in the vertebroplasty group $(8 \%, n=7)$ : 


\begin{tabular}{|c|c|c|c|}
\hline \multirow[b]{2}{*}{ Time points } & \multicolumn{3}{|c|}{ Mean VAS scores $(95 \% \mathrm{Cl})^{\star}$} \\
\hline & Vertebroplasty $(n=90)$ & Sham procedure $(n=86)$ & Group differencet \\
\hline 1 day & $5.24(4.73$ to 5.76$)$ & $4.82(4.29$ to 5.34$)$ & $-0.43(-1.17$ to 0.31$)$ \\
\hline 1 week & $4.38(3.86$ to 4.90$)$ & $4.27(3.74$ to 4.79$)$ & $-0.11(-0.85$ to 0.63$)$ \\
\hline 1 month & $3.32(2.80$ to 3.84$)$ & $3.73(3.20$ to 4.26$)$ & $0.41(-0.33$ to 1.15$)$ \\
\hline 6 months & $3.02(2.48$ to 3.55$)$ & 3.41 (2.86 to 3.96$)$ & $0.39(-0.37$ to 1.15$)$ \\
\hline 12 months & $2.72(2.18$ to 3.26$)$ & $3.17(2.60$ to 3.75$)$ & $0.45(-0.37$ to 1.24$)$ \\
\hline Difference between baseline and 12 months & $5.00(4.31$ to 5.70$)$ & $4.75(3.93$ to 5.57$)$ & $0.13(-0.41$ to 0.66$)$ \\
\hline \multicolumn{4}{|c|}{$\begin{array}{l}\text { VAS scores range from } 0 \text { (no pain) to } 10 \text { (worst pain), with } 1.5 \text { designated as the minimally clinically important difference. Model estimates were adjusted } \\
\text { based on age, sex, vertebral level, pain treatment, osteonecrosis, new fractures after baseline, and progressive loss of vertebral height. } \\
\text { *Statistically significant difference between VAS score at baseline and each follow-up. } \\
\text { tBetween group differences are contrast estimates at each time point based on custom hypothesis test function in SPSS mixed analysis. Positive } \\
\text { estimates favour the sham procedure, negative estimates favour vertebroplasty. }\end{array}$} \\
\hline
\end{tabular}

$\left(\chi^{2}(1)=25.85, \mathrm{P}<0.001\right)$. At the 12 month follow-up, participants with progressive loss of vertebral height had significantly higher VAS scores (4.20, 95\% confidence interval 3.44 to 5.06 ) than participants without loss of vertebral height $(2.76,2.32$ to 3.20). These differences, however, were not affected by whether participants received vertebroplasty or the sham procedure.

A third non-prespecified analysis showed that during the 12 months' follow-up, 31 new fractures occurred in 15 participants in the vertebroplasty group and 28 in 19 participants in the sham procedure group. The occurrence of new vertebral fractures did not differ significantly between the two groups $\left(\chi^{2}(1)=0.83\right.$, $\mathrm{P}=0.36$, odds ratio 0.71 , $95 \%$ confidence interval 0.33 to 1.50 ). Twelve participants (six in each group) underwent re-intervention during follow-up for one or more new symptomatic fractures. All were treated according to the original assignment.

Use of analgesics decreased statistically significantly in both groups for all three drug categories (non-opioid, weak opioid, and strong opioid) at all time points with

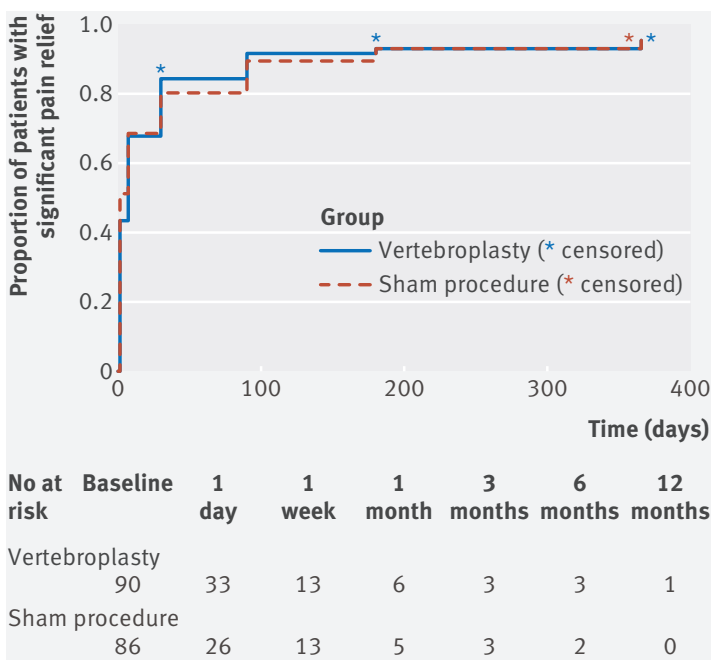

Fig 3 | Kaplan-Meier survival curves for time to clinically significant pain relief (decrease of 1.5 points on visual analogue scale) from baseline in vertebroplasty and sham procedure groups. Patients who were lost to follow-up before assessment of pain relief were censored no statistically significant differences between the groups. Appendix table A3 and figure A1 show the reduction in analgesic use over the 12 month study period, with the largest reduction between baseline and one month. At baseline, 13 participants in the vertebroplasty group $(14.4 \%$, 95\% confidence interval $7.0 \%$ to $21.8 \%$ ) and 17 in the sham procedure group $(19.8 \%, 11.2 \%$ to $28.4 \%)$ were using weak opioids. At one month this usage had decreased to six participants $(7 \%, 1.4 \%$ to $12.6 \%)$ in the vertebroplasty group and four $(4.7 \%, 0.1 \%$ to $9.3 \%)$ in the sham procedure group. This reduction in weak opiate use did not differ significantly between the two groups $(7.7 \%$, 95\% confidence interval $-5.0 \%$ to $20.4 \%$ ).

At baseline, 42 participants in the vertebroplasty group $(46.7 \%, 36.2 \%$ to $57.2 \%)$ and 25 in the sham procedure group $(29 \%, 19.2 \%$ to $38.8 \%)$ were using strong opioids. At one month this usage had decreased to 18 participants in the vertebroplasty group $(20.9 \%$, $11.6 \%$ to $30.2 \%$ ) and 19 in the sham procedure group $(22.4 \%, 13.4 \%$ to $31.4 \%)$. This reduction in strong opiate use was significantly larger in the vertebroplasty group (19.2\%, 1.8\% to 36.6\%). Both groups showed no statistically significant reduction of analgesic use at three, six, and 12 months compared with one month measures.

The mean volume of cement used in participants in the vertebroplasty group was 5.11 (SD 1.81) $\mathrm{mL}$ (range 1-11 mL). Computed tomography performed on the same day as the procedure showed evidence of cement leakage in 105 of the 115 treated vertebral bodies (91\%). See appendix table A4 for further details of cement leakage.

Two adverse reactions occurred, both in the vertebroplasty group. One participant with severe chronic obstructive pulmonary disease developed respiratory insufficiency the day after the procedure, related to underlying pulmonary disease. Another participant had a vasovagal reaction during the procedure that spontaneously resolved.

\section{Discussion}

Our results show that in patients with acute osteoporotic compression fractures of the vertebral body who have persistent severe local back pain, 
percutaneous vertebroplasty performed at a mean of 43 days after onset of symptoms did not result in greater pain relief than a sham procedure during 12 months' follow-up. The sham intervention comprised local infiltration with $1 \%$ lidocaine (lignocaine) followed by $0.25 \%$ bupivacaine into each pedicle and simulated cement (polymethylmethacrylate) injection with verbal and physical cues. Participants in both study groups showed immediate improvement in pain and disability after the procedure, and this improvement was sustained during 12 months' follow-up. These results suggest that factors aside from instillation of polymethylmethacrylate might have accounted for the observed clinical improvement after vertebroplasty; for example, the effect of local anaesthesia, expectations of pain relief (placebo effect), natural healing of the fracture, and regression to the mean.

\section{Comparison with previous studies}

This trial reinforces the results of two previous blinded trials $^{56}$ and the Cochrane review about percutaneous vertebroplasty, ${ }^{19}$ with the added value of longer followup, larger cohorts, and stricter inclusion criteria-that is, only acute fractures with bone oedemain participants with visual analogue scale (VAS) scores of 5 or higher. In contrast with the Investigational Vertebroplasty Safety and Efficacy Trial (INVEST) by Kallmes and colleagues, the Australian trial by Buchbinder and colleagues, ${ }^{56}$ and the current study (VERTOS IV), the results from the VERTOS II and VAPOUR trial favoured vertebroplasty. ${ }^{78}$ Even though, like the three blinded trials, the VAPOUR trial is a blinded randomised controlled trial, many differences exist with the former randomised controlled trials. Most noticeably, VAPOUR included predominantly hospital inpatients (56$58 \%$ ) with pain for less than six weeks (mean 2.4-2.8 weeks), high VAS scores ( $>7)$ at baseline necessitating opiate analgesics in $90 \%$ of participants, and a sham procedure without infiltration of the pedicle. In line with VAPOUR, the unblinded randomised clinical trial VERTOS II found a statistically significant advantage in the vertebroplasty group, including pain reduction, compared with conservative treatment. Inherent in the unblinded design is the potential overestimation of treatment benefit that results from an uneven placebo response between groups. ${ }^{7}$

\section{Strengths and weaknesses of this study}

Most pain relief in both groups of the unblinded VERTOS II and this blinded study occurred during the first month, with a sustained slow decrease in both groups throughout follow-up. In the current study, however, clinically significant pain relief was achieved earlier in the sham procedure group (after one day) than in the vertebroplasty group (after one week). The Kaplan-Meier survival analysis indicated no statistically significant differences between the groups in the progress of pain relief. The use of analgesics decreased statistically significantly for participants in both groups, with the largest improvement during the first month, but without statistically significant differences between groups for pain and class of analgesics.

The present study showed a positive treatment effect, with improvement of both RMDQ and QUALEFFO scores but with no statistically significant difference between groups. The measured RMDQ scores for disability measured at one month were similar to those observed in INVEST. ${ }^{5}$ Compared with the Australian trial, both groups in this study showed a substantially better treatment effect, with a higher improvement of VAS, RMDQ, and QUALEFFO scores during the first six months. ${ }^{6}$ This difference might be because the Australian trial only included a small percentage of participants with acute vertebral fracture (32\%) who had symptoms for less than six weeks before treatment, whereas in this study the participants were treated at a median of six weeks after onset of symptoms.

More than $80 \%$ of the participants in the sham procedure group of the current study believed that they had received cement compared with $37 \%$ in INVEST $^{5}$ and $54 \%$ in VAPOUR. ${ }^{8}$ This suggests a greater placebo effect in the sham procedure group of VERTOS IV. Despite spontaneous pain reduction from fracture healing and the placebo effect that might contribute to the observed treatment effect, ${ }^{20}$ ${ }^{21}$ we did not anticipate the magnitude of the positive treatment response in the sham procedure group. In a Cochrane review on placebo studies the effect of placebo on pain varied from negligible to clinically important. $^{22}$ Another study noted that a placebo effect is related to treatment context, including the physician's attitude and patient's expectations. ${ }^{23}$ These factors might have played a strong part in the positive response of participants in the sham procedure group. This trial utilised the same periosteal local anaesthetic regimen as INVEST, whereas VAPOUR only infiltrated the subcutaneous space. Periosteal infiltration using local anaesthetic might have a treatment effect and this could perhaps partially explain the observed difference in response of the sham procedure group.

This study has several limitations. The trial lacked an additional control group that received standard medical conservative treatment. We did not compare the study groups for other medical treatments that might have affected outcomes. The persistence of pain after intervention or sham intervention might indicate causes other than a fracture, such as osseous or disc degenerative disease, a possibility depicted by magnetic resonance imaging, but this was not meticulously analysed. Overall, 156 patients declined participation, conferring a generalisability problem given that most chose to undergo vertebroplasty. No screening logs were kept for these patients. This can suggest a selection bias towards recruitment of patients with lesser degrees of pain and disability. During the trial we extended the pain window from six to nine weeks, a potential source of selection bias. The statistical analysis and writing of the manuscript were not done blinded and could be sources of bias. 
The study has limited generalisability to other forms of augmentation or vertebral treatments, such as instrumented vertebroplasty or balloon or radiofrequency kyphoplasty and patients with chronic or malignant vertebral fractures.

We performed three post hoc observations during follow-up. A statistically significant higher proportion of participants with VAS scores of 5 or higher at 12 month follow-up were noticed after the sham procedure compared with vertebroplasty ( $41 \%$ v 20\%). During the 12 months' follow-up imaging showed a statistically significant higher incidence of further vertebral body collapse after the sham procedure. ${ }^{78}$ This confirms the assumption that cementation protects against progressive collapse of the vertebral body.

The use of polymethylmethacrylate showed no effect on the development of new fractures at adjacent or remote levels. ${ }^{724}$ The results of VERTOS II and the current study show that the cause of adjacent level and remote fractures are most likely the result of the generalised osteoporotic disease and the osteoporotic spine and not related to cementation. To identify and define the predictive factors at baseline responsible for these post hoc outcomes (patients with considerable persistent pain (VAS score $\geq 5$ ) and progressive loss of vertebral body height, at 12 months) further research is warranted. It is not inconceivable or discounted that this subgroup can be identified and distinguished at baseline as those patients with acute osteoporotic vertebral compression fractures suitable for early intervention.

\section{Implications and future research}

According to this study vertebroplasty in the early phase of a painful osteoporotic vertebral compression fracture does not provide important pain relief compared with a sham procedure. The results suggest that periosteal infiltration alone in the early phase provides enough pain relief with no need for additional cementation. In the late phase of a painful vertebral compression fracture, there seems to be a subgroup of conservatively treated patients who still experience substantial local back pain at 12 months' follow-up. Predictors are still not identified and are considered important subjects for future investigation.

Despite the outcome of this trial, we continue to offer vertebroplasty to a proportion of referred patients. We believe there is a place for vertebroplasty when efficacy outweighs the risks. The lower and upper limits of reasonable applicability of vertebroplasty are shown more or less by the results of previous trials, 5625 suggesting a window in which vertebroplasty might outperform conventional treatment. In general, if vertebroplasty is performed too early then treatment will largely be for fractures that are destined to improve anyway by natural healing. If vertebroplasty is performed after many months of pain, the treatment will largely be for fractures that are no longer responsive. Thus, a future therapeutic pain strategy could be a combined regimen of periosteal infiltration during natural healing. Additional cementation seems indicated only in a selected subgroup of patients with insufficient pain relief after this early phase. In our opinion, this strategy provides clinicians with a pragmatic approach on how to best treat patients. However, according to the results of VAPOUR, there might be an important exception to this rule for a subgroup of patients-that is, hospital inpatients with more comorbidity and high VAS scores $(>7)$ due to an acute osteoporotic vertebral compression fracture. In this subgroup of patients, cementation at an early stage ( $<6$ weeks) might be justified. Research is warranted to prove that vertebroplasty is better than periosteal infiltration of local anaesthetic in hospital inpatients, since this has never been investigated.

\section{Conclusion}

Percutaneous vertebroplasty did not result in statistically significant greater pain relief than a sham procedure during 12 months follow-up among a group of patients with acute osteoporotic compression fractures of the vertebral body. These results do not support using percutaneous vertebroplasty to treat acute osteoporotic vertebral compression fractures.

We thank Lieke Lalieu and Dennis Carli (Elisabeth TweeSteden Hospital), Femmy Meenhorst (Albert Schweitzer Hospital), Bas Pierik (Medisch Spectrum Twente), and Marion Blonk, Jerôme Kisters, Irene Horst, Monique Vos, and Riekie van Beers (Catharina Hospital Eindhoven)

Contributors: CEF and PNML had full access to all the data in the study, take responsibility for the integrity of the data and the accuracy of the data analysis, and act as guarantors. PNML, CAHK, WJJVR, JdV, MCS, AJS, JRJ, ED, OEHE, FHJ, and AVT designed the study. IB and KS gathered the data. PNML, MCS, AJS, CAHK, OEHE, FHJ, and AVT performed the procedures. PL and JdV analysed the data. CEF and AV undertook morphometric measurements. CEF, PNML, AV, JdV, PL, and JAH wrote the first draft, and all the contributors made the decision to submit the manuscript for publication.

Funding: This study was supported by Stryker (grant No S-1-013). The sponsor had no role in the design and conduct of the study; collection, management, analysis, and interpretation of the data; preparation, review, or approval of the manuscript; and decision to submit the manuscript for publication

Competing interests: All authors have completed the ICMJE uniform disclosure form at www.icmje.org/coi_disclosure.pdf and declare: no support from any organisation for the submitted work; no financial relationships with any organisations that might have an interest in the submitted work in the previous three years; no other relationships or activities that could appear to have influenced the submitted work. JAH has received consulting fees from Medtronic and Globus as well as serving on a data and safety monitoring board of a study sponsored by Codman Neurovascular.

Ethical approval: This trial was approved by the institutional review boards of the four participating hospitals (MN-11-004, approval protocol 1055).

Data sharing: Dataset available from the corresponding author at crisfiranescu@hotmail.com.

Transparency: The study guarantors (CEF and PNML) affirm that the manuscript is an honest, accurate, and transparent account of the study being reported; that no important aspects of the study have been omitted; and that any discrepancies from the study as planned have been explained.

This is an Open Access article distributed in accordance with the Creative Commons Attribution Non Commercial (CC BY-NC 4.0) license, which permits others to distribute, remix, adapt, build upon this work non-commercially, and license their derivative works on different terms, provided the original work is properly cited and the use is noncommercial. See: http://creativecommons.org/licenses/by-nc/4.0/.

1 Jha RM, Yoo AJ, Hirsch AE, Growney M, Hirsch JA. Predictors of successful palliation of compression fractures with vertebral augmentation: single-center experience of 525 cases. / Vasc Interv Radiol 2009;20:760-8. doi:10.1016/j.jvir.2009.01.037 
2 Voormolen MH, Lohle PN, Lampmann LE, et al. Prospective clinical follow-up after percutaneous vertebroplasty in patients with painful osteoporotic vertebral compression fractures. J Vasc Interv Radiol 2006;17:1313-20. doi:10.1097/01.RVI.0000231952.75209.4A

3 Anselmetti GC, Manca A, Hirsch J, et al. Percutaneous vertebroplasty in osteoporotic patients: an institutional experience of 1,634 patients with long-term follow-up. J Vasc Interv Radiol 2011;22:1714-20. doi:10.1016/j.jvir.2011.09.001

4 Barr JD, Jensen ME, Hirsch JA, et al. Position Statement on Percutaneous Vertebral Augmentation: A Consensus Statement. Developed by the American Association of Neurological Surgeons(AANS) and the Congress of Neurological Surgeons (CNS), American College of Radiology (ACR), American Society of Neuroradiology (ASNR), American Society of Spine Radiology (ASSR), Canadian Interventional Radiology Association (CIRA), Society of Interventional Radiology (SIR), and the Society of Neurolnterventional Surgery (SNIS). J Vasc Interv Radiol 2014;25:171-81. doi:10.1016/j. jvir.2013.10.001

5 Kallmes DF, Comstock BA, Heagerty PJ, et al. A randomized trial of vertebroplasty for osteoporotic spinal fractures. N Engl J Med 2009;361:569-79. doi:10.1056/NEJMoa0900563

6 Buchbinder R, Osborne RH, Ebeling PR, et al. A randomized trial of vertebroplasty for painful osteoporotic vertebral fractures. $N$ Engl Med 2009;361:557-68. doi:10.1056/NEJMoa0900429

7 Klazen CA, Lohle PN, de Vries J, et al. Vertebroplasty versus conservative treatment in acute osteoporotic vertebral compression fractures (Vertos II): an open-label randomised trial. Lancet 2010;376:1085-92. doi:10.1016/S01406736(10)60954-3

8 Clark W, Bird P, Gonski P, et al. Safety and efficacy of vertebroplasty for acute painful osteoporotic fractures (VAPOUR): a multicentre, randomised, double-blind, placebocontrolled trial. Lancet 2016;388:1408-16. doi:10.1016/S01406736(16)31341-1

9 Firanescu C, Lohle PN, de Vries J, et al. VERTOS IV study group. A randomised sham controlled trial of vertebroplasty for painful acute osteoporotic vertebral fractures (VERTOS IV). Trials 2011;1745:12-93.

10 McHugh GA, Luker KA, Campbell M, Kay PR, Silman AJ. A longitudinal study exploring pain control, treatment and service provision for individuals with end-stage lower limb osteoarthritis. Rheumatology (Oxford) 2007;46:631-7. doi:10.1093/rheumatology/kel355

11 Genant HK, Wu CY, van Kuijk C, Nevitt MC. Vertebral fracture assessment using a semiquantitative technique. J Bone Miner Res 1993;8:1137-48. doi:10.1002/jbmr.5650080915

12 Huskisson EC. Measurement of pain. Lancet 1974:2:1127-31. doi:10.1016/S0140-6736(74)90884-8

13 Lips P, Cooper C, Agnusdei D, et al, Working Party for Quality of Life of the European Foundation for Osteoporosis. Quality of life in patients with vertebral fractures: validation of the Quality of Life Questionnaire of the European Foundation for Osteoporosis (QUALEFFO). Osteoporos Int 1999;10:150-60. doi:10.1007/s001980050210
14 Patrick DL, Deyo RA, Atlas SJ, Singer DE, Chapin A, Keller RB. Assessing health-related quality of life in patients with sciatica. Spine (Phila Pa 1976) 1995;20:1899-908, discussion 1909. doi:10.1097/00007632-199509000-00011

15 Black DM, Cummings SR, Karpf DB, et al, Fracture Intervention Trial Research Group. Randomised trial of effect of alendronate on risk of fracture in women with existing vertebral fractures. Lancet 1996;348:1535-41. doi:10.1016/S0140-6736(96)07088-2

16 R Development Core Team. R: A language and environment for statistical computing. R Foundation for Statistical Computing, Vienna, Austria. ISBN 3-900051-07-0. 2008. http://www.R-project.org.

17 Grotle M, Brox JI, Vøllestad NK. Concurrent comparison of responsiveness in pain and functional status measurements used for patients with low back pain. Spine (Phila Pa 1976) 2004;29: E492-501. doi:10.1097/01.brs.0000143664.02702.0b

18 Ostelo RW, Deyo RA, Stratford P, et al. Interpreting change scores for pain and functional status in low back pain: towards international consensus regarding minimal important change. Spine (Phila Pa 1976) 2008:33:90-4. doi:10.1097/BRS.0b013e31815e3a10

19 Buchbinder R, Golmohammadi K, Johnston RV, et al. Percutaneous vertebroplasty for osteoporotic vertebral compression fracture. Cochrane Database Syst Rev 2015;(4):CD006349. doi:10.1002/14651858.CD006349.pub2.

20 Venmans A, Klazen CA, Lohle PN, Mali WP, van Rooij WJ. Natural history of pain in patients with conservatively treated osteoporotic vertebral compression fractures: results from VERTOS II. A/NR Am I Neuroradiol 2012;33:519-21. doi:10.3174/ajnr.A2817

21 Krogsbøll LT, Hróbjartsson A, Gøtzsche PC. Spontaneous improvement in randomised clinical trials: meta-analysis of three-armed trials comparing no treatment, placebo and active intervention. BMC Med Res Methodol 2009;9:1. doi:10.1186/1471-2288-9-1

22 Hróbjartsson A, Gøtzsche PC. Placebo interventions for all clinical conditions. Cochrane Database Syst Rev 2010:20:CD003974.

23 Manchikanti L, Pampati V, Hirsch JA. Analysis of utilization patterns of vertebroplasty and kyphoplasty in the Medicare population. / Neurointerv Surg 2013;5:467-72. doi:10.1136/ neurintsurg-2012-010337

24 Uppin AA, Hirsch JA, Centenera LV, Pfiefer BA, Pazianos AG, Choi IS. Occurrence of new vertebral body fracture after percutaneous vertebroplasty in patients with osteoporosis. Radiology 2003;226:119-24. doi:10.1148/radiol.2261011911

25 Rousing R, Hansen KL, Andersen MO, Jespersen SM, Thomsen K. Lauritsen IM. Twelve-months follow-up in forty-nine patients with acute/semiacute osteoporotic vertebral fractures treated conservatively or with percutaneous vertebroplasty: a clinical randomized study. Spine (Phila Pa 1976) 2010;35:478-82. doi:10.1097/BRS.0b013e3181b71bd1

Supplementary information: tables A1-A4 and figure A1 